\title{
Mobile Phone Addiction and Sleep Quality in Chinese College Students
}

\author{
Fu Guifang ${ }^{1}$, Sun Chenxia ${ }^{1}$, Ding Ruijia ${ }^{2 *}$ \\ ${ }^{I}$ Department of Applied Psyghology, Guangdong University of Foreign Studies, Canton, China \\ ${ }^{2}$ College of Education, University of Washington, Seattle, Washington, USA
}

*Corresponding Author: Ding Ruijia, College of Education, University of Washington, Seattle, Washington, USA

\begin{abstract}
With the popularity of mobile phones, the positive and negative effects of mobile phone use are increasing. Smartphones bring a lot of convenience to people, but they also seriously affect people's physical and mental health. Mobile Phone Addiction (MPA) has become a public health issue. This study explored the status and relationship of Chinese college students' mobile phone addiction and quality of sleep in order to improve the quality of sleep of college students. This study was conducted in universities in Guangdong province in China. Using the Smartphone Addiction Scale(SAS) and the Pittsburgh Sleep Quality Index(PSQI), 250 Chinese college students were surveyed on mobile phone addiction and sleep quality through the Internet.The results showed that college students' mobile phone addiction was at a moderate level, and 31.6\% of them had sleep problem. Female students were more addicted to mobile phone than boys, but there is no gender difference in sleep quality.There was a significant positive correlation between cell phone addiction and sleep quality. Cell phone addiction could predict $14.8 \%$ of sleep quality.College Students' mobile phone addiction and sleep problems are more serious, and the more serious cell phone addiction, the more serious sleep problems. Future research should focus on the intervention of cell phone addiction.
\end{abstract}

Keywords: college student; mobile phone addiction; sleep quality

\section{INTRODUCTION}

Mobile phone addiction (MPA) has become a public health issue. With the popularity of mobile phones, the positive and negative effects of mobile phone are increasing. The positive role of mobile phone is mainly to make communication more convenient, fast and optimized. People can access various services such as banking, booking, shopping, even health management and sports management and so on through mobile phones. However, the negative impact of mobile phones is also very obvious, mainly including mobile phone addiction, sleep problem, financial problems, lack of concentration, delay, time management problem, even personal safety and cyber bullying etc. Mobile phone addiction has become more and more harmful to health, especially the impact on sleep. Therefore, this study attempts to explore the current status of mobile phone addiction and its relationship with sleep quality in college students.

Studies have shown that with the accelerated pace of modern social life, college students' emotional adjustment difficulties, self social avoidance and distress are all related to emotional adjustment ability and cell phone addiction(Zhu, 2015). Mobile phone addiction is usually targeted at various network functions on mobile phones, such as social networks, online games, online fiction, online shopping, online sexual behavior, etc. and it has a high degree of overlap and similarity with internet addiction (Fu, Jin\&Guo, 2019). Leung (2008) etc. suggested that there are four characteristics of mobile phone addiction: out of control, abstinence, avoidance and inefficiency. Out of control means inability to control craving to use moblie phone. Abstinence means the frustration caused by the failure to meet the needs of mobile phones. Avoidance refers to the use of mobile phones to avoid negative emotions or real pressures. Inefficiency means the reduction of learning and working efficiency caused by improper use of mobile phones (Leung, 2008; Liu, Zhou\&Niu, 2017. Fu etc. (2019) found that $36.77 \% \%$ of Chinese college students had serious mobile phone addiction and that 
girls were significantly higher than boys in mobile phone addiction and out of control of its subdimension.

Sleep quality ( SQ ) is a comprehensive evaluation of sleep processes and effects, in addition to objective sleep conditions, including subjective sleep experience(Zhou, 2010).The decline in sleep quality has become one of the most striking health problems in modern society. More and more modern people cannot guarantee high quality sleep due to various external or internal factors. Sleep problems affect human social function and cognitive function, and are also early warning signals for various diseases. Sleep quality might have a greater impact on subjective cognitive decline (SCD), and SCD-related functional difficulties in younger adults, compared with in older adults(Lee, Ju, Park \&Lee, 2020). A study of college students in 26 countries found that sleep quality was closely related to social demographic variables, health risk behaviors and health index. The sleep quality of college students is attracting attention from all walks of life, so this study has strong theoretical and practical value.

\section{METHOD}

\subsection{Participants and Procedure}

A cross-sectional survey was conducted through a professional online platform what is named Wenjuanxing (Questionnaire star)for data collection questionnaire survey from January to February 2018. A convenience cluster sampling method was employed to produce a sample of college students. All participants were recruited from Guangdong province in south China. Everybody involved in this study will be told to participant voluntarily and could withdraw at any time. 332 Chinese college students were tested and 250 valid questionnaires were returned. Among them there were 68 males and 182 females and aged between 17 and 23, with an average of 21.16 years old.

\subsection{Measures}

Smartphone Addiction Scale (SAS). This study used the Smartphone Addiction Scale (SAS) compiled by Kwon and Lee (2013). The scale contains a total of 33 items. Items are scored on a 6-point Likert scale ranging from 1 (very disagree) to 6 (very agree). The total score of SAS ranges from 33 to 198. Higher scores indicated higher levels of mobile phone addiction. The Cronbach's alpha in this study was 0.967.

Pittsburgh Sleep Quality Index (PSQI).This work used Pittsburgh Sleep Quality Index (PSQI) compiled by Buysse et al for testing sleep quality in nearly one month (Buysse\&Reynolds, 1989). The questionnaire consisted of 19 questions, including seven factors: subjective sleep quality (SSQ), time to fall asleep (TFA), sleep duration (SD), sleep efficiency (SE), sleep disorder (SD), sleep drug use (SDU) and daytime function barriers (DFB). SD refers to insomnia, excessive sleepiness, sleepdisordered breathing, and abnormal sleep behavior caused by various causes. DFB refers to daytime fatigue, drowsiness, snoring, poor energy, unresponsiveness, decreased judgment, confusion, and mental disorders. Each factor is scored on a scale of 0-3, and finally the scores of the seven factors are added to obtain a total score of PSQI. The total score range of PSQI in this work is 0 to 21 , and the higher the score, the worse the sleep quality. In this study, the total score was $\geq 8$ for the detection of sleep disorders, and the single factor score $\geq 2$ indicates that the quality or quantity of the factor was poor or poor(He, Chen \&Bao, 2012)The Cronbach's alpha of PSQI was 0.83.

\subsection{Data Analysis}

The descriptive analysis was used to determine the demographic characteristics of the participants. Pearson correlation analyses and linear regression of the study variables were conducted. We adopt independent-samples T-test to examine differences in mobile phone addiction and sleep quality between. Statistical analysis was conducted using the SPSS 17.0 version program.

\section{ReSUlts}

\subsection{The Relationship between MPA and SQ}

Descriptive statistics showed that the average score of SAS is 121.97. The total score of SAS is 198. It indicated that college students' mobile phone addiction is at a medium level. The average of sleep quality was 6.20 and the maximum value is 21 . Except for sleep drug use, all other dimensions of 
sleep quality were positively correlated with cell phone addiction. To determine whether or not mobile phone addiction predicted sleep quality, standard multiple regression analysis was performed. This model explained $14.8 \%$ of the variances in sleep quality scores $\left(R^{2}=0.151\right.$, adjusted $R^{2}=0.148$, $\mathrm{p}=0.000)$.

Table1: Descriptive statistics of MPA and its correlation with $S Q, M P A$ as predictor $S Q$

\begin{tabular}{|l|l|l|l|}
\hline & \multicolumn{1}{|c|}{$\begin{array}{c}\text { M } \pm \text { MPD } \\
\text { MPA }\end{array}$} & \multicolumn{1}{|c|}{$\mathbf{R}^{2}$} & \multicolumn{1}{|c|}{${\text { Adjusted } \mathbf{R}^{2}}$} \\
\hline MPA & $121.97 \pm 25.05$ & .151 & .148 \\
\hline SQ & $6.20 \pm 3.15 .389^{* *}$ & & \\
\hline Subjective Sleep Quality & $.227^{* *}$ & & \\
\hline Time to Fall Asleep & $.290^{* *}$ & & \\
\hline Sleep Duration & $.195^{* *}$ & & \\
\hline Sleep Efficiency & $.210^{* *}$ & & \\
\hline Sleep Disorder & $.291^{* *}$ & & \\
\hline Sleep Drug Use & .085 & & \\
\hline Daytime Function Barriers & $.289^{* *}$ & & \\
\hline
\end{tabular}

**p $<0.01$,SD, standard deviation, MPA, mobile phone addiction, SQ, Sleep Quality

This study found that $31.6 \%$ of college students had sleep problems (PSQI total score $>8$ ), of which $30.4 \%$ had subjective sleep quality problems, $43.6 \%$ had time to fall asleep, $15.6 \%$ had duration problems, $18.8 \%$ had sleep efficiency problems, $20.4 \%$ had sleep disorder, $4.4 \%$ had sleep medication problems, and $28.4 \%$ had daytime function problems. It can be seen that nearly one-third of contemporary college students have poor quality of sleep, especially the time required to fall asleep, daytime function barriers and subjective sleep quality problems.

\subsection{Comparison for Mobile Phone Addiction and Sleep Quality by Gender}

Independent sample t-test results showed there were gender differences in college students' mobile phone addiction, and girls were more serious. In general, there was no gender difference in the quality of sleep among college students, but boys' sleep duration was more serious than girls' and girls' daytime dysfunction was more serious than boys'.

Table2: Gender Differences of $M P A$ and $S Q$

\begin{tabular}{|l|l|l|l|l|}
\hline & \multicolumn{1}{|c|}{$\begin{array}{c}\text { Male }(\mathbf{n = 6 8}) \\
\mathbf{M} \pm \text { SD }\end{array}$} & $\begin{array}{c}\text { Female }(\mathbf{n = 1 8 2}) \\
\mathbf{M} \pm \text { SD }\end{array}$ & \multicolumn{1}{c|}{$\boldsymbol{p}$} & \multicolumn{1}{c|}{} \\
\hline MPA & $115.60 \pm 23.66$ & $124.35 \pm 25.20$ & $-2.483^{*}$ & 0.014 \\
\hline SQ & $6.04 \pm 3.31$ & $6.26 \pm 3.10$ & -0.477 & 0.634 \\
\hline SSQ & $1.24 \pm 0.77$ & $1.19 \pm 0.74$ & 0.405 & 0.686 \\
\hline TFA & $1.38 \pm 0.95$ & $1.44 \pm 0.95$ & -0.425 & 0.672 \\
\hline SD & $0.82 \pm 0.81$ & $0.56 \pm 0.75$ & $2.423^{*}$ & 0.016 \\
\hline SE & $0.49 \pm 0.86$ & $0.60 \pm 0.92$ & -0.884 & 0.377 \\
\hline SD & $1.03 \pm 0.60$ & $1.13 \pm 0.62$ & -1.179 & 0.240 \\
\hline SMU & $0.16 \pm 0.54$ & $0.13 \pm 0.54$ & 0.464 & 0.643 \\
\hline DFB & $0.93 \pm 0.72$ & $1.20 \pm 0.71$ & $-2.731^{* *}$ & 0.007 \\
\hline
\end{tabular}

*p $<0.05, * * p<0.01$, subjective sleep quality (SSQ), time to fall asleep(TFA), sleep duration(SD), sleep efficiency(SE), sleep disorder(SD), sleep drug use(SDU) and daytime function barriers(DFB)

\subsection{Comparison for Sleep Quality by Mobile Phone Addiction}

According to the score of mobile phone addiction scale from low to high, the top $30 \%$ were non-cell phone addicts, and the last $30 \%$ were mobile phone addiction group. The results showed that there were significant differences in total sleep quality and its 7 specific dimensions between the mobile phone addiction group and the non-addiction group. The sleep quality of all the students in the non mobile phone addiction group was significantly better than those in the mobile phone addiction group.

Table3: Sleep differences in mobile phone addiction

\begin{tabular}{|l|c|c|c|c|}
\hline & $\begin{array}{c}\text { MPA } \\
\text { M } \pm \text { SD }\end{array}$ & $\begin{array}{c}\text { Non-MPA } \\
\text { M } \pm \text { SD }\end{array}$ & $\boldsymbol{t}$ & \multicolumn{1}{c|}{} \\
\hline SQ & $7.69 \pm 3.53$ & $5.08 \pm 2.66$ & $-5.093^{* *}$ & 0.000 \\
\hline
\end{tabular}




\begin{tabular}{|l|l|l|l|l|}
\hline SSQ & $1.39 \pm 0.84$ & $1.07 \pm 0.74$ & $-2.503^{*}$ & 0.013 \\
\hline TFA & $1.73 \pm 1.00$ & $1.17 \pm 0.94$ & $-3.513^{* *}$ & 0.001 \\
\hline SD & $0.81 \pm 0.89$ & $0.51 \pm 0.70$ & $-2.320^{*}$ & 0.022 \\
\hline SE & $0.88 \pm 1.02$ & $0.48 \pm 0.83$ & $-2.616^{* *}$ & 0.010 \\
\hline SD & $1.28 \pm 0.69$ & $0.93 \pm 0.53$ & $-3.468^{* *}$ & 0.001 \\
\hline SMU & $0.18 \pm 0.60$ & $0.01 \pm 0.12$ & $-2.268^{*}$ & 0.026 \\
\hline DFB & $1.40 \pm 0.70$ & $0.91 \pm 0.66$ & $-4.469^{*}$ & 0.000 \\
\hline
\end{tabular}

$* \mathrm{p}<0.05, * * \mathrm{p}<0.01$

\section{DisCUSSIONS}

\subsection{College Students' Mobile Phone Addiction is at a Medium Level}

Results of this study showed that the average score of mobile phone addiction among college students reached 121.97, while the score of this scale ranged between 33 and 198, which was higher than the average score under the standard normal distribution (115.5), indicating that the problem of mobile phone addiction in Chinese college students was serious. Results were consistent with previous findings of the probability of mobile phone addiction in college students (Fu, Jin, \&Guo, 2019; , Xie, Chen, Zhu, \&He,2019;Tingting Gao etc. 2018, Songli Mei etc.2018).

Nowadays, mobile phones are becoming more and more powerful. People's work and life are more and more inseparable from mobile phones. As a young fashion leader, college students will first try new functions of mobile phones, such as mobile phone payment, mobile shopping, mobile phone reading and so on. At the same time, college students have more free time, which leads to more time for college students to use mobile phones, and it can easily lead to cell phone addiction various kinds of Internet addiction. Research found that $62.8 \%$ of college students were addicted to online shopping (Zhang, Bai, Wei, Yang, \& Fu, 2019). In addition, college students are in the period of perfecting selfidentity and establishing intimate relationship with others. They are prone to psychological conflicts and negative emotions. Mobile phones are the most convenient way to release negative emotions, which also increases the possibility of mobile phone addiction among college students.

\subsection{About One Third of College Students Have Sleep Problems}

This study suggested that the average score of PSQI of college students was 6.20 which is consistent with previous studies (Liu, Zhou, \&Niu, 2017; Ling \& Xin, 2014; Liu, Zhou, Yang, Kong, Niu, \&Fan, 2017). Liu etc. also found that average score of PSQI of Chinese adolescent was 6.31 in 2017. Taking the total score of PSQI $\geq 8$ as the detection criterion, this study found that the rate of sleep disorders among college students was as high as $31.6 \%$, which was significantly higher than the previous 1520\% (Zhang\&Diao, 2006; Zou, Zou, \&Yao, 2011; Li, Mei, Niu, \&Song,2016). It can be seen that the sleep problem of college students is becoming more and more serious. A study showed that $20 \%$ Japanese young adult' sleep quality was poor (Naoki Toyama et al., 2019).

\subsection{Mobile Phone Addiction is Closely Related to Sleep Quality}

This study found that there was a significant positive correlation between cell phone addiction and sleep quality, which is consistent with previous studies (Liu, Zhou, Yang, Kong, Niu, \&Fan,2017; Li, Mei, Niu, \&Song, 2016). This study not only confirmed conclusions of previous studies, but also further found that college students' mobile phone addiction had more and more influence on sleep quality. Demirci K and others showed that depression, anxiety and sleep quality may be related to the excessive use of smart phones (Demirci, Akgönül, \& Akpinar, 2006). And Feng et al also found that the length of the subjects watching the mobile phone screen was significantly related to the quality of sleep (Feng, Zhang, Qing, Du, Ye, \&He, 2014). Zou Yunfei's research on college students also suggested that the proportion of mobile phone addicts in sleep quality was significantly higher than that of non mobile phone addicts (2011). Liu Qingqi and others (2017) also found that cell phone addiction has a direct predictive effect on the sleep quality of adolescents. So future research should focus on how to intervene in cell phone addiction in order to improve sleep quality.

\subsection{Limitations}

There are some confound factors in this study, which was based on a self-reported questionnaire online. On the one hand, online questionnaires cannot control interference variables very well. On the other hand, cross-sectional research cannot get causal relationship. Therefore, we should further develop mobile phone dependence longitudinal cross lagging research and experimental research in 
the future. Finally, future studies should further explore other factors affecting sleep quality and increase sample size of the survey.

\section{CONCLUSION}

College Students' mobile phone addiction and sleep problems are more serious, and the more serious cell phone addiction, the more serious sleep problems. Future research should focus on the intervention of cell phone addiction.

\section{ACKNOWLEDGEMENT}

This work was supported by The National Social Science Fund of China [grant number 16BSH094].

\section{REFERENCES}

[1] China Internet Network Information Center.(2018).The 41st Statistical Report on Internet Development in China.http://www.sohu.com/a/220271269_582307

[2] Daniel J. Buysse, Charles F. Reynolds, Timothy H. Monk, Susan R. Berman, David J. Kupfer. (1989).The Pittsburgh Sleep Quality Index: a new instrument for psychiatric practice and research. Psychiatry research, 28(2): 193-213.https://doi.org/10.1016/0165-1781(89)90047-4.

[3] Demirci K, Akgönül M, Akpinar A.(2015).Relationship of smartphone use severity with sleep quality, depression, and anxiety in university students. Journal of behavioral addictions, 4(2): 85-92. DOI:10.15 56/2006.4.2015.010

[4] Feng, Qi; Zhang, Qing-le; Du, Yue; Ye, Yong-ling; He, Qi-qiang.(2014).Associations of physical activity, screen time with depression, anxiety and sleep quality among Chinese college freshmen. PloS One, 9(6): 1-5. DOI: 10.1371/journal.pone.0100914

[5] Fu Guifang, Jin Yuting, Guo Jia. (2019).Mobile Phone Addiction and Career Preparation in College Students ,Higher Education of Social Science.16(2):10-16 DOI: 10.3968/11215

[6] He Jinbo, Chen Changrun, Bao Yuanchun.(2012).Measurement, harm and mechanism of mobile phone dependence in adolescents. Chinese Journal of Clinical Psychology. 20(6): 822-825. DOI : 10.16128/j. cnki.1005-3611.

[7] JooEun Lee, Yeong Jun Ju, Eun-Cheol Park, Soon Young Lee, (2019).Effect of poor sleep quality on subjective cognitive decline (SCD) or SCD-related functional difficulties: Results from 220,000 nationwide general populations without dementia, Journal of Affective Disorders. 2020; 260:32-37, https:// doi.org/10.1016/j.jad.

[8] Kwon Min, Kim Dai-Jin, Cho Hyun, Yang Soo.(2013).Development and validation of a smartphone addiction scale (SAS). PloS one, 8(2): DOI: 10.1371/journal.pone.0083558

[9] Leung L. (2008).Linking psychological attributes to addiction and improper use of the mobile phone among adolescents in Hong Kong. Journal of Child Media. 2: 93-113.

[10] Li Li, Mei Songli, Niu Zhimin, Song Yuting.(2016).The relationship between loneliness and sleepquality incollege students: the mediating role of smartphone addiction and the role of gender regulation. Chinese Journal of Clinical Psychology.24(2): 345-348. DOI : 10.16128/j.cnki.1005-3611.

[11] Ling Huanxi, Xin Ziqiang.(2014).A study of the traversal history of college students' sleep quality changes. Chinese Journal of Mental Health.28(10): 786-790.

[12] Liu Qingqi, Zhou Zongkui, Niu Gengfeng.(2017).Mobile phone addiction and adolescent sleep quality: analysis of mediation and regulation. Acta Psychologica Sinica.49(12): 1524-1536.

[13] Naoki Toyama, Daisuke Ekuni, Ayano Taniguchi-Tabata, Toshiki Yoneda, Kota Kataoka, Aya Yokoi, Yoko Uchida, Daiki Fukuhara, Hikari Saho, Islam Md Monirul, Nanami Sawada, Yukiho

[14] Nakashima, Yoshiaki Iwasaki, Manabu Morita.(2019)Associations between sleep bruxism, sleepquality, and exposure to secondhand smoke in Japanese young adults: a cross-sectional study, Sleep Medicine, in press, https://doi.org/10.1016/j.sleep.2019.09.003.

[15] Qing-Qi Liu, Zong-Kui Zhou, Xiu-Juan Yang, Fan-Chang Kong, Geng-Feng Niu, Cui-Ying

[16] Fan,(2017).Mobile phone addiction and sleep quality among Chinese adolescents: A moderated mediation model,Computers in Human Behavior, 72,108-114 https://doi.org/10.1016/j.chb.2017.02.042

[17] Songli Mei, Gang Xu, Tingting Gao, Hui Ren ,Jingyang Li.(2018).The relationship between college students' alexithymia and mobile phone addiction: Testing mediation and moderation effects. $B M C$ Psychiatry.18:329 https://doi.org/10.1186/s12888-018-1891-8

[18] Tingting Gao, Jiaomeng Li, Han Zhang, Jinglei Gao, Yixi Kong, Yueyang Hu, Songli Mei.(2017).The influence of alexithymia on mobile phone addiction: The role of depression, anxiety and stress. Journal of Affective Disorders, 2018; 225:761-766 http://dx.doi.org/10.1016/j.jad. 
[19] Xiaochun Xie, Wu Chen, Xiaowei Zhu, Dan He.(2019).Parents' phubbing increases Adolescents' Mobile phone addiction: Roles of parent-child attachment, deviant peers, and gender. Children and Youth Services Review,105, https://doi.org/10.1016/j.childyouth.2019.104426

[20] Zhang Jiahui, Bai Zhiqiang, Wei Jingxia, Yang Maolin\& Fu Guifang.(2019).The Status Quo of College Students' Online Shopping Addiction and Its Coping Strategies International Journal of Psychological Studies, Vol. 11, No. 2, 88-93, https://doi.org/10.5539/ijps.v11n2p88

[21] Zhang Lin, Diao Juan. (2016).Study on sleep quality and related factors of college students. Chinese Journal of Clinical Psychology.14(5): 515-517. DOI : 10.16128/j.cnki.1005-3611.2006.05.028

[22] Zhou Xihua.(2010).Research on College Students' Mobile Phone Addiction. Education and Teaching Research. 24(4): 16-18, 35.

[23] ZouYunfei, Zou Yunqing, Yao Yingshui.(2011).A cross-sectional survey of mobile phone use and mobilephone dependence in college students. Journal of Gannan Medical College.30 (1): 77-80.

[24] Zhu Yuxi. (2015).Relationship between mobile phone addiction and sleep quality in college students. Social Psychology. (10): 73-80

Citation: Ding Ruijia, et.al. "Mobile Phone Addiction and Sleep Quality in Chinese College Students". International Journal of Humanities Social Sciences and Education (IJHSSE), vol. 6, no.11, 2019, pp. 25-30. doi: http://dx.doi.org/10.20431/2349-0381.0611004.

Copyright: (C) 2019 Authors. This is an open-access article distributed under the terms of the Creative Commons Attribution License, which permits unrestricted use, distribution, and reproduction in any medium, provided the original author and source are credited. 